\title{
Upgrading Pantograph Performance Using Variable Stiffness Devices
}

\author{
Yoshitaka YAMASHITA, Dr. Eng. \\ Assistant Senior Researcher, \\ Mitsuru IKEDA \\ Laboratory Head, \\ Current Collection Laboratory, Railway Dynamics Division
}

\begin{abstract}
This paper introduces a new technique for improving pantograph current collection performance. The technique involves replacing pan springs with variable stiffness devices. The device described here comprises two facing air springs. This paper first gives an outline of the method used for improving current collection performance together with some results from numerical simulations, which demonstrate the technique's effectiveness. Secondly the paper briefly introduces a prototype of the variable stiffness device, describing its basic characteristics. Finally, results are presented from experiments in controlling dynamic pantograph characteristics using the variable stiffness devices.
\end{abstract}

Keywords: pantograph, variable stiffness device, current collection

\section{Introduction}

The pantograph is the device through which electric power is collected and supplied to a railway vehicle. Positioned on the roof of vehicles they come into direct contact with overhead contact lines. They are therefore subject to various disturbances such as contact forces from overhead contact wires as well as frictional and aerodynamic forces. As a result, there is a risk of contact loss between the pantograph and overhead lines or of excessive upward force being exerted by the pantograph onto the contact wire. Contact loss can lead to arcing which causes damage both to the pantograph and overhead contact lines. Excessive upward force exerted by the pantograph can also cause damage to overhead lines such as local wear or breakage. To avoid this type of damage and ensure that pantograph uplift is optimal, contact forces should be kept as uniform as possible. As such contact forces are seen as an index of the dynamic interaction in the overhead contact line/pantograph system. Literature already exists for research on reducing contact force fluctuation by means of actively controlled pantographs (e.g. [1-5]). However it is still necessary to simplify the complicated pantograph/catenary system model to apply the above active control techniques with high contact performance. The limited space around the pantograph is another obstacle since it is not always possible to actually use the desired actuation system. For instance, applying a control force to the base frame is easier than directly to the pantograph head or contact strip. The frame actuation has a filtering effect on actions aimed at the pantograph head [2]. It is hard to find an actuator small enough to be installed directly below the pantograph head which also has enough power to control the contact force. As such there are still many difficulties to overcome before being able to apply active control techniques to pantographs.

Meanwhile, the contact performance of a pantograph is often evaluated in terms of compliance characteristics. The compliance characteristics specify when a pantograph runs along an overhead rigid conductor having sinusoidal irregularities, the maximum amplitude of overhead rigid conductor irregularities with which the pantograph can run without causing a contact loss. Compliance characteristics can be calculated on the basis of frequency response, defined as static uplift force of a pantograph multiplied by a transfer function between contact forces and pantograph head displacement. The results usually produce a set of frequencies which show where compliance characteristics peak or trough. At the peak frequency, contact force fluctuation is usually smaller than that at the other frequencies. These peak and trough frequencies are primarily determined by the masses used in a pantograph dynamic model and/or the stiffness of the spring components placed in between the masses.

In this study, we focused on identified compliance characteristics and proposed a new technique for improving pantograph performance by controlling compliance characteristics through varying the spring stiffness.

\section{A new technique for improving pantograph performance}

A typical pantograph configuration is as follows: Figure 1(a) is a mechanical diagram example of a pantograph, based on a double mass model as shown in Fig.1(b). The raising mechanism located in the base frame ensures a constant uplift force to the articulated frame consisting of a lower and an upper frame. Raising mechanisms of many pantographs comprises a coil spring and a lever. The lever ratio changes in relation to the articulated frame height so that the uplift force produced by deformation of the coil spring can be maintained almost constant. Parallel to this, is a pantograph damper. The pan springs are located at the top of the upper frame and support the pantograph head. The contact strips which are in direct contact with overhead line or rigid conductor are fixed to the pantograph head.

In this pantograph model, the pantograph head and the contact strips are expressed as a single mass element $m_{1}$. The upper and the lower frames are also simplified as a single mass element $m_{2}$. Displacements of these masses are expressed as $x_{1}$ and $x_{2}$, respectively. The two masses 
are connected by a pan spring $k_{1}$. The damping element $c_{1}$ located between the two masses is also represented. $c_{2}$ and $F_{0}$ describe the damping coefficient of the pantograph damper and the constant uplift force produced by the raising mechanism. The pantograph is subject to a fluctuating contact force $f_{C}$, from overhead rigid conductors or contact wires.

Based on the dynamic model of the pantograph shown in Fig.1 (b), the corresponding equation of motion is:

$$
\left\{\begin{array}{c}
m_{1} \ddot{x}_{1}+c_{1}\left(\dot{x}_{1}-\dot{x}_{2}\right)+k_{1}\left(x_{1}-x_{2}\right)=-f_{C} \\
m_{2} \ddot{x}_{2}+c_{2} \dot{x}_{2}+c_{1}\left(\dot{x}_{2}-\dot{x}_{1}\right)+k_{1}\left(x_{2}-x_{1}\right)=F_{0}
\end{array}\right.
$$

The transfer function defined by the ratio between the contact force and pantograph head displacement is:

$$
\frac{X_{1}(s)}{F_{C}(s)}=\frac{\left\{m_{2} s^{2}+\left(c_{1}+c_{2}\right) s+k_{1}\right\}}{\left(m_{1} s^{2}+c_{1} s+k_{1}\right)\left\{m_{2} s^{2}+\left(c_{1}+c_{2}\right) s+k_{1}\right\}-\left(c_{1} s+k_{1}\right)^{2}}
$$

where $X_{1}(s)$ and $F_{C}(s)$ are the Laplace transforms of $x_{1}$ and $f_{C}$. Compliance characteristics are given as follows:

$$
H(\omega)=\left|X_{1}(i \omega) / F_{C}(i \omega)\right| F_{0} .
$$

Examples compliance characteristics curves are shown in Fig.2. The curves are obtained by substituting the three different $k_{1}$ values and parameters listed in Table 1 into (3). Results usually show that the different $k_{1}$ produce peaks at the different frequencies.

If the stiffness of the pan spring can be varied continuously, the peak frequency for the compliance characteristics curve may be set arbitrarily. The bold line in Fig.2 indicates the trajectory of the peak point of the curves as stiffness of the pan spring is varied.

When the overhead rigid conductor has sinusoidal irregularities, the pantograph passing along the conductor experiences a sinusoidal forced displacement disturbance of frequency $f_{W}[\mathrm{~Hz}]$ which is determined by the running speed of the pantograph $V[\mathrm{~m} / \mathrm{s}]$ and the wave length of the sinusoidal irregularities of the conductor $L$ $[\mathrm{m}]$ as:

$$
f_{W}=V / L \text {. }
$$

Let us consider the case where stiffness of the pan spring is variable, and where a pantograph equipped with such a pan spring is running along a rigid conductor with sinusoidal irregularities. When frequency $f_{W}$ is consistent

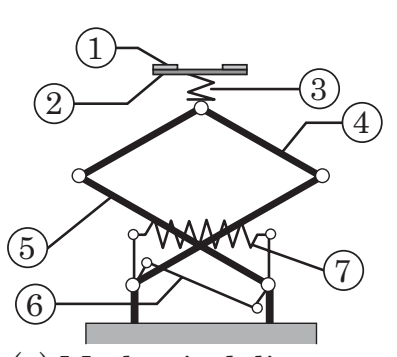

(a) Mechanical diagram

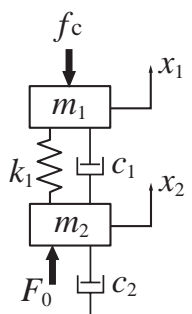

W

(b) Dynamic model
Fig. 1 Mechanical diagram and dynamic model of a pantograph

1: Contact strip, 2: Pantograph head, 3: Pan spring,

4: Upper frame, 5: Lower frame, 6: Balancing rod, 7: Raising mechanism \& pantograph damper

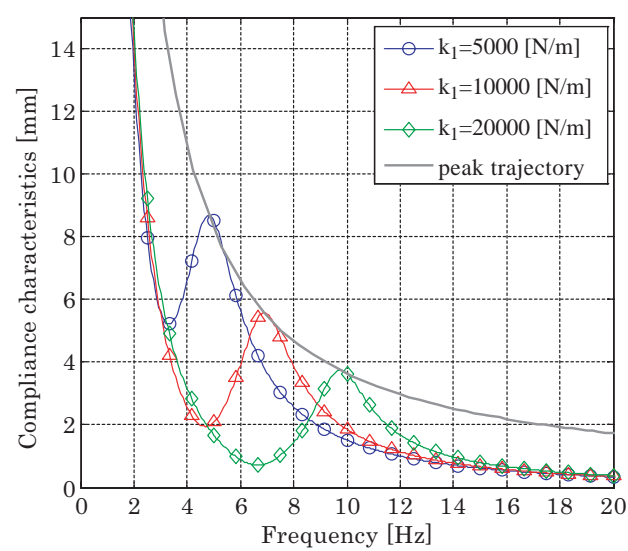

Fig. 2 Compliance characteristics

Table 1 Pantograph specification

\begin{tabular}{|c|c|}
\hline Parameters & Values \\
\hline$m_{1}[\mathrm{~kg}]$ & 10.3 \\
\hline$m_{2}[\mathrm{~kg}]$ & 11.2 \\
\hline$c_{1}[\mathrm{Ns} / \mathrm{m}]$ & 50 \\
\hline$c_{2}[\mathrm{Ns} / \mathrm{m}]$ & 80 \\
\hline$P_{0}[\mathrm{~N}]$ & 54 \\
\hline
\end{tabular}

with the trough frequency of the compliance characteristics curve, contact performance falls. In such a case, contact performance may be improved by controlling stiffness of the pan spring to achieve consistency between the compliance characteristics' peak frequency and the disturbance frequency. In this paper, we propose a new technique for improving pantograph contact performance by controlling the pan spring stiffness based on the abovementioned discussion. This technique is less complicated than the active control techniques.

\section{Numerical simulation}

In the previous chapter, a new technique for improving pantograph performance was introduced. However, when we calculate the compliance characteristics, we assume that the pantograph equipped with the variable stiffness device is running along a rigid overhead conductor with sinusoidal irregularities. That is not always the case on actual commercial lines today because many lines have overhead catenary system.

In this chapter, the technique presented above is therefore applied to a catenary system and numerical simulations were used to verify the new technique. Figure 3 briefly describes the pantograph running simulation. Two pantographs, one equipped with constant stiffness springs and the other with variable stiffness devices ran along the overhead catenary system over five spans. One pantograph runs in each simulation, and the results from the pantographs equipped with constant and variable stiffness springs are compared. The running speed of the pantograph varied between $40 \mathrm{~km} / \mathrm{h}$ to $280 \mathrm{~km} / \mathrm{h}$. A compound catenary system was used as overhead line equipment for these tests, comprising three wires termed 
as messenger wire, auxiliary messenger wire and contact wire from on high. Table 2 lists the primary parameters of the equipment. The three wires were fixed at both the ends. The messenger wires were also fixed at the supporting points. Rigid rods were used as droppers and hangers which were fixed only to the lower wire and suspended from the upper wire. In this simulation, floating of droppers and hangers from the upper wire due to pantograph passage was taken into account. The three wires were modeled using a discrete mass model, and the masses interconnected by a spring and damper. All the masses including the pantograph masses were only able to move vertically. This simulation also used the pantograph model shown in Fig. 1 (b) and the parameters listed in Table 1. In case of the constant stiffness pan spring, $k_{1}=14700$ $[\mathrm{N} / \mathrm{m}]$.

In order to control the stiffness of the variable stiffness device, it is necessary to determine a target frequency, which is consistent with the compliance characteristics peak frequency. It is well-known that the dominant contact wire irregularities are due to dips between the hangers which excite pantograph head motion. The dominant wave length for the irregularities therefore is hanger span $L_{h}$. The target frequency $f_{\text {target }}$, i.e. the dominant disturbance frequency to the pantograph, is:

$$
f_{\text {target }}=V / L_{h} \text {. }
$$

Note that the dominant wave length can be equal to dropper or supporting point span rather than hanger span. In such a case, we just have to set the $L_{h}$ value in (5) to be dropper or supporting point span.

Figure 4 indicates stiffness for constant and variable stiffness pan springs as a function of the running speed. The stiffness of the variable stiffness pan spring increases with running speed. The compliance characteristics for the pantograph equipped with the variable stiffness de- vice are shown in Fig.5. For convenience, the corresponding speed, used in (5), is given below the frequency axis. In the case of a pantograph equipped with a constant stiffness pan spring frequency on the compliance characteristics curves always peaks at around $8.3 \mathrm{~Hz}$, which corresponds to a target frequency of running speed of $150 \mathrm{~km} /$ $h$. The contact performance of pantographs with the constant and the variable stiffness devices is evaluated in this simulation by looking at the contact force between the pantograph head and contact wire. Standard deviations of the contact forces of the pantographs running through the $3^{\text {rd }}$ and $4^{\text {th }}$ spans are compared to evaluate the contact performance of the constant and variable stiffness pan spring at each running speed. This contact performance comparison is illustrated in Fig.6. For speeds of $200 \mathrm{~km} / \mathrm{h}$ and above the variable stiffness pan spring outperforms the constant pan spring. This is because in such cases the dominant contact force frequencies are consistent with the target frequencies in the high speed range. On the other hand, for speeds below $180 \mathrm{~km} / \mathrm{h}$, it is hard to distinguish a difference in performance. At lower

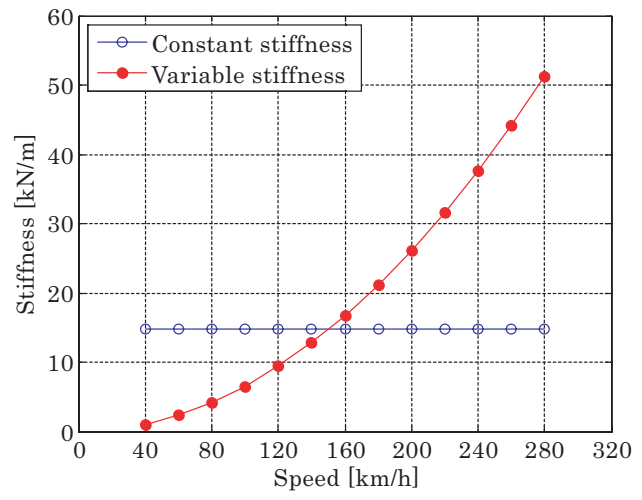

Fig. 4 Relation of pan spring stiffness to running speed

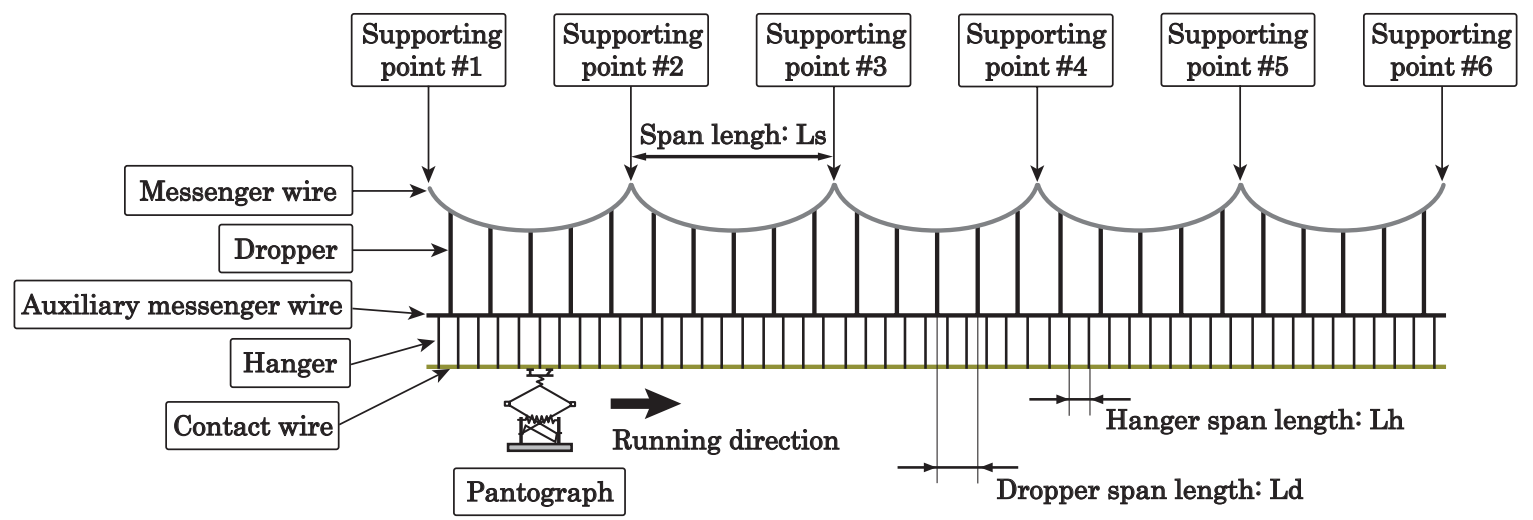

Fig. 3 Schematic description of pantograph running simulation

Table 2 Compound catenary equipment specification

\begin{tabular}{|c|c|c|c|}
\hline Span length, $L_{s}[\mathrm{~m}]$ & \multicolumn{3}{|c|}{50} \\
\hline Dropper span length, $L_{d}[\mathrm{~m}]$ & \multicolumn{3}{|c|}{10} \\
\hline Hanger span length, $L_{h}[\mathrm{~m}]$ & \multicolumn{3}{|c|}{5} \\
\hline & Messenger wire & Auxiliary messenger wire & Contact wire \\
\hline Tension $[\mathrm{N}]$ & 24500 & 14700 & 14700 \\
\hline Line density $[\mathrm{kg} / \mathrm{m}]$ & 1.450 & 1.375 & 1.511 \\
\hline Wave propagation velocity $[\mathrm{km} / \mathrm{h}]$ & 468.0 & 372.2 & 355.1 \\
\hline
\end{tabular}




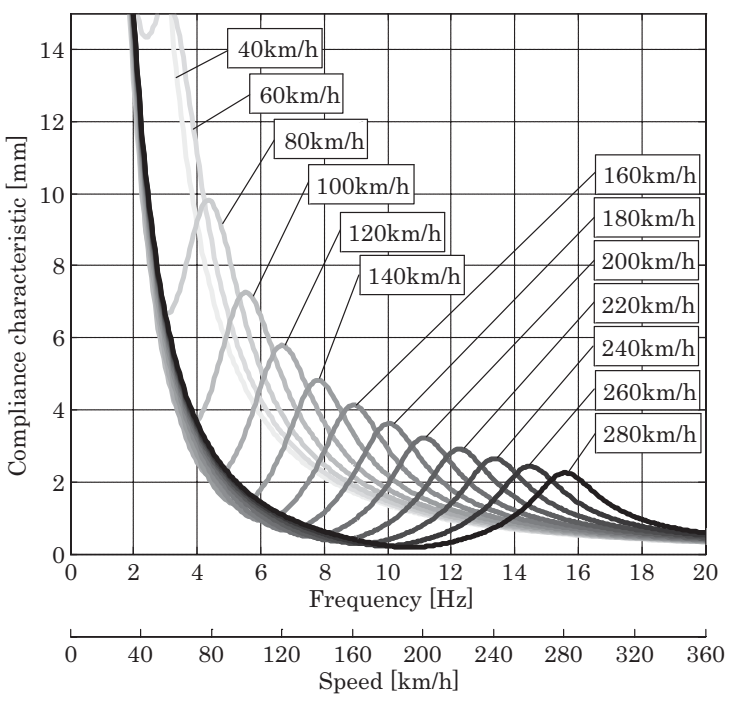

Fig. 5 Compliance characteristics of the variable stiffness device equipped pantograph

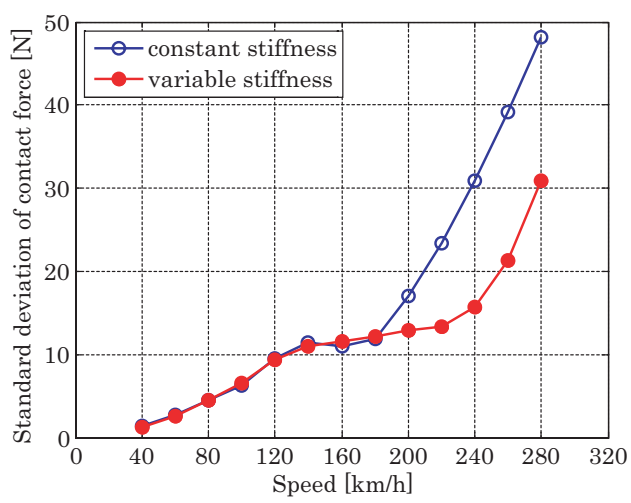

Fig. 6 Standard deviation of contact forces as a function of running speed

speeds it is apparent that the dominant contact force frequencies are different from the target frequencies [6]. The results from lower speed simulations imply that improvement in contact performance using the variable stiffness pan spring can only be achieved when target frequency and dominant frequency of contact force fluctuation are consistent. Although from all the simulation results, we confirmed that the variable stiffness pan springs do not worsen the contact performance.

\section{Variable stiffness device}

This chapter introduces the prototype of variable stiffness device. A photograph and diagram of the device are shown in Fig.7. It is composed of two facing air springs. Pressure resistance of the device is approximately 0.20 $\mathrm{MPa}$. Note that the term pressure used throughout this paper refers to the difference with atmospheric pressure. One end of each air spring is fixed, while the other end is connected to the mobile part of the variable stiffness device. External forces to the device are transmitted to this mobile part.

Let us assume pressure and volume of $P_{0}$ and $V_{0}$ in both the air springs when the device is in the neutral position $(x=0)$ shown in Fig.7 (b). Compressed air of pressure $P_{0}$ is first injected into both the air springs via the pneumatic lines. A stop valve then serves to seal the air chamber on each air spring. Let us then consider the situation where the mobile part is displaced by $\mathrm{x}$ due to an external force $F$. Motion in the mobile part causes variations in the pressure and the volume in each chamber.

In Fig.7, the pressure and volume of the lower air spring changes from $P_{0}$ to $P_{1}\left(>P_{0}\right)$ and $V_{0}$ to $V_{1}\left(<V_{0}\right)$, respectively. The corresponding change in the upper air spring is from $P_{0}$ to $P_{2}\left(<P_{0}\right)$ and $V_{0}$ to $V_{2}\left(>V_{0}\right)$, respectively. On the assumption that the state of the air in each chamber changes polytropically during displacement of the mobile part and that chamber volume varies linearly with respect to the displacement of the moving part, one obtains:

$$
P_{0} V_{0}^{\gamma}=P_{1} V_{1}^{\gamma}=P_{2} V_{2}^{\gamma},
$$

and

$$
\left\{\begin{array}{l}
V_{1}=V_{0}-A x \\
V_{2}=V_{0}+A x
\end{array},\right.
$$

where $A$ is the constant, which in this study is the pressure receiving area, and $\gamma$ is the polytropic index. If the force is in equilibrium, one can obtain:

$$
F=A\left(P_{1}-P_{2}\right),
$$

From (6) to (8), the relationship between the displacement and the restoring force of this variable stiffness device is expressed as:

$$
F=A P_{0}\left\{\left(\frac{V_{0}}{V_{0}-A x}\right)^{\gamma}-\left(\frac{V_{0}}{V_{0}+A x}\right)^{\gamma}\right\} .
$$

After performing a first order Taylor series expansion around $x=0$, one can obtain the following relationship:

$$
F=\frac{2 \gamma A^{2} P_{0}}{V_{0}} x
$$

The coefficient of $x$ is the stiffness of the device, which is defined at the neutral position. The device stiffness obtained by varying the neutral pressure $P_{0}$ is arbitrary.

In order to investigate how the relationship between displacement and restoring force of the device depends on the level of pressure in the air springs at neutral position, the mobile part of the device is subjected to a quasistatic forced displacement with the air springs set at different neutral pressures. The frequency and displacement amplitude input are $0.1 \mathrm{~Hz}$ and $5 \mathrm{~mm}$, respectively. Figure 8 shows the relationship between the displacement and the restoring force of the device for the neutral pressures set at $0.00 \mathrm{MPa}, 0.05 \mathrm{MPa}, 0.10 \mathrm{MPa}, 0.15 \mathrm{MPa}$ and $0.20 \mathrm{MPa}$. Applying the least square method to each experimental curve in Fig.8, produces the equivalent linear stiffness of the device. Figure 9 expresses the stiffness of the device as a function of the neutral pressure. It can be seen that device stiffness increases with neutral pressure. Equation (10) describes this linear relationship, however, in practice this relationship appears nonlinear. This is supposed to be because the higher order term in (10) is neglected and because of assumptions such as the receiv- 


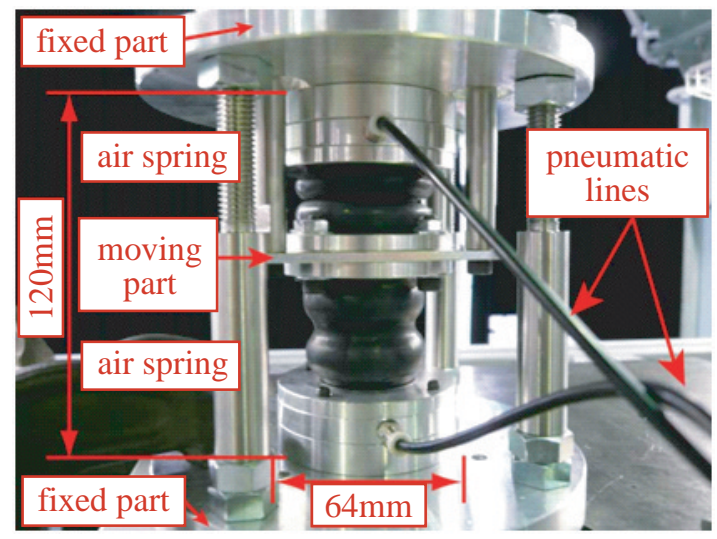

(a) Picture

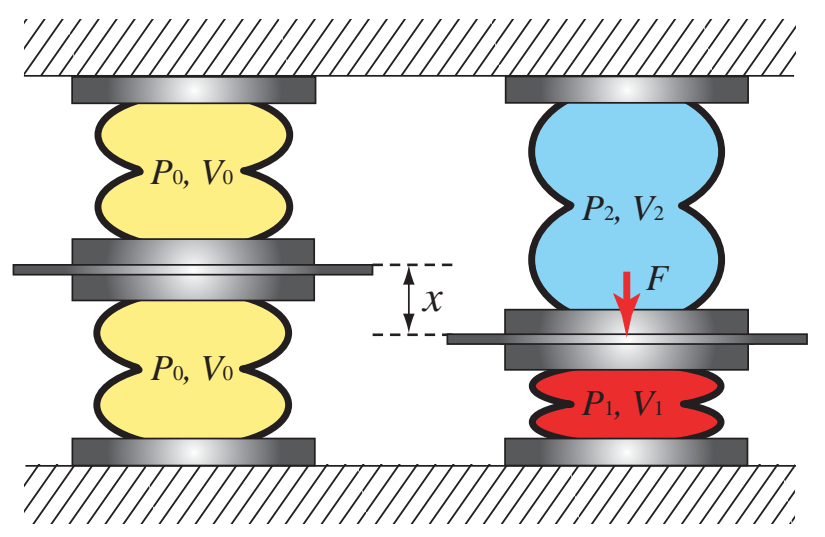

(b) Schematic diagram

Fig. 7 Variable stiffness device

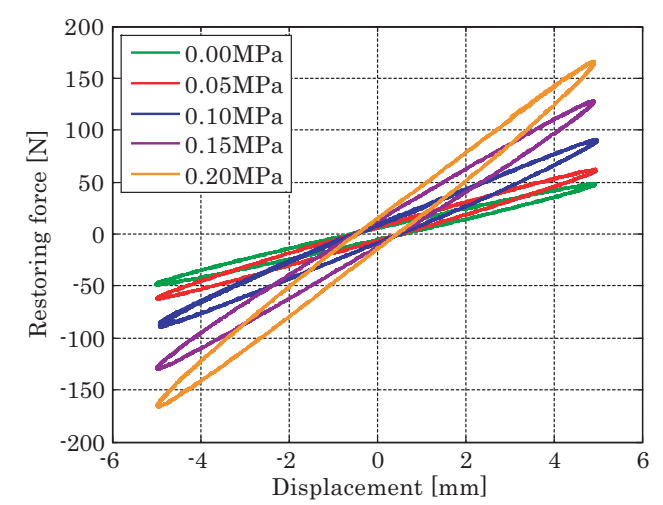

Fig. 8 Influence of the pressure on the relationship between displacement and restoring force of the variable stiffness device

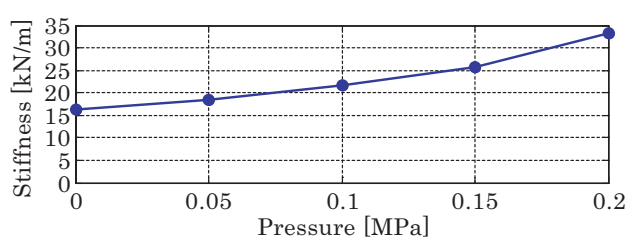

Fig. 9 Relationship between pressure and stiffness of the variable stiffness device

ing area being constant or the linear dependence of the chamber volume on displacement of mobile parts or to geometrical nonlinearity due to the inflation of rubber parts.

\section{Controlling the dynamic characteristics of the pantograph}

With a view to controlling the dynamic characteristics of the pantograph, the pan springs are replaced with the variable stiffness device introduced in former chapter. Figure 10 shows the experimental setup of the pantograph equipped with the variable stiffness device. Two variable stiffness devices are installed on this pantograph to test the possibility of the compliance characteristics control. The pantograph is settled below the vibration exciter, and the pantograph head and vibration exciter are rigidly connected. The vibration exciter moves vertically to subject the pantograph to pseudo-random forced displacement vibration. The input displacement contains the frequency components of up to $32 \mathrm{~Hz}$. The contact force between the pantograph head and the vibration exciter is measured by a load cell located between them. Pantograph head displacement, which is equal to the input displacement, is measured by the displacement sensor embedded in the vibration exciter. In order to evaluate the contact performance of this pantograph, contact force $f_{C}$ and pantograph head displacement $x_{1}$ data acquired during the time series are converted into frequency series, and the compliance is calculated. The compliance is defined by:

$$
G(\omega)=\left|X_{1}(i \omega) / F_{C}(i \omega)\right| .
$$

Note that the relationship between compliance and the compliance characteristics of the pantograph is as follows:

$$
H(\omega)=G(\omega) F_{0} .
$$

Figure 11 exhibits the compliance of the pantograph equipped with a variable stiffness device. The neutral pressure of the devices is set to $0.00 \mathrm{MPa}, 0.10 \mathrm{MPa}$ and $0.20 \mathrm{MP}$. The peak frequency can be shifted by means of varying the neutral pressures of the devices. From these results, we can conclude that it is possible to control the pantograph compliance characteristics by using the variable stiffness device described in this paper.

The variable stiffness device presented in this paper can vary the stiffness from approximately $16200 \mathrm{~N} / \mathrm{m}$ to $33200 \mathrm{~N} / \mathrm{m}$ (see Fig.9) so that the total stiffness of this pantograph can be varied from approximately $32400 \mathrm{~N} / \mathrm{m}$ to $66400 \mathrm{~N} / \mathrm{m}$, and the corresponding peak compliance frequency varies between about $15 \mathrm{~Hz}$ to $20 \mathrm{~Hz}$.

Compliance in relation to the neutral pressure of the variable stiffness devices may be roughly estimated using the pantograph's parameters (e.g. table 1) and the dependence of stiffness on pressure as shown in Fig.9. Figure 12 shows compliance calculated on the basis of stiffness and pantograph parameters and on the basis of direct measurement. The calculated and measured results are represented on the graph by solid lines and markers, respectively. The latter results coincide exactly with those shown in Fig.11 but are down-sampled for comparison with the calculated results. The calculated results are more or less in line with the measured results except at the neutral pressure of $0.20 \mathrm{MPa}$. In future it would cer- 


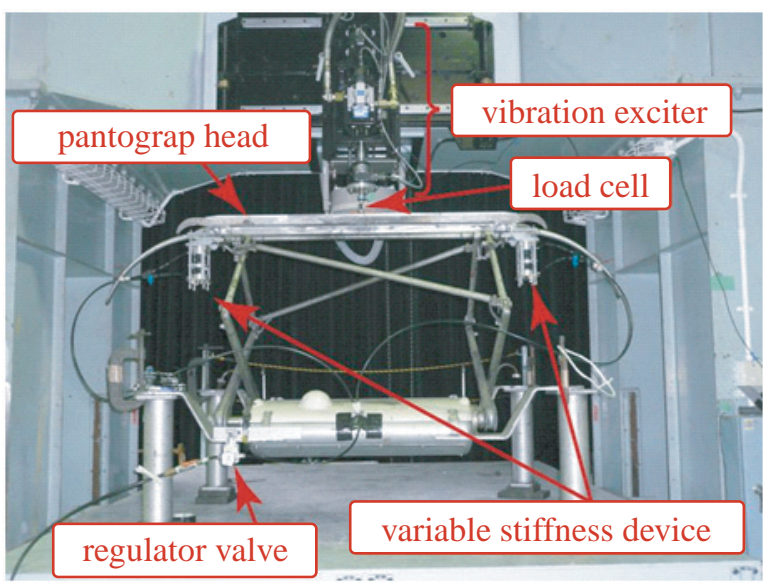

(a) Overall view

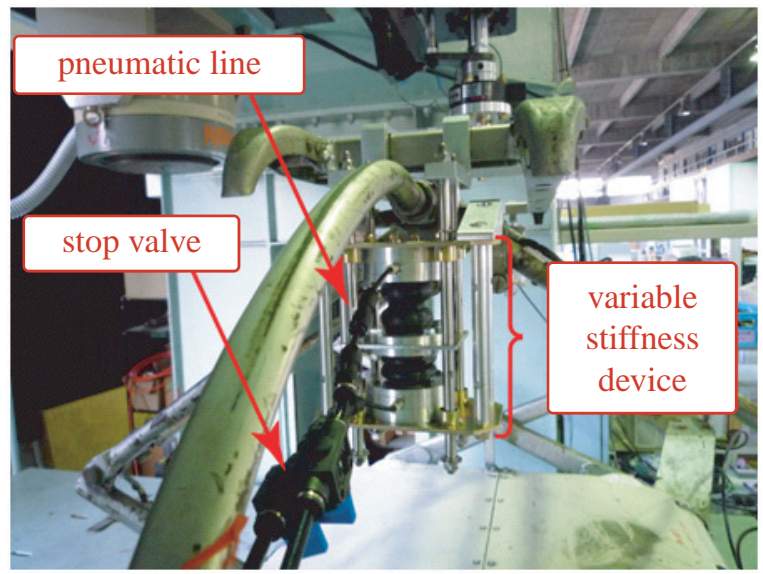

(b) Detail

Fig. 10 Pictures of a pantograph equipped with a variable stiffness device

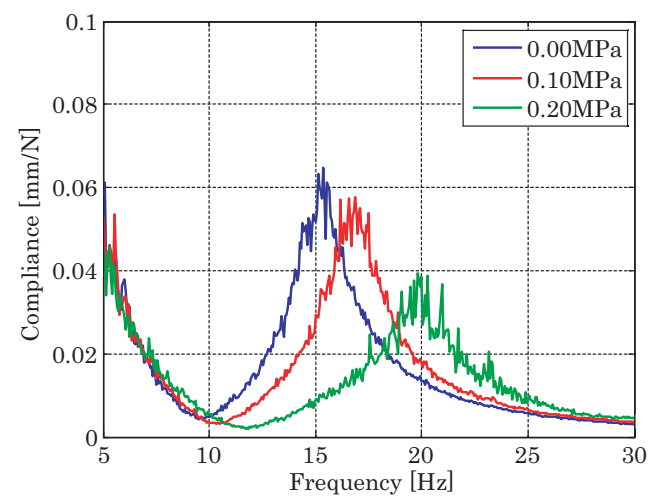

Fig. 11 Compliance control of the pantograph by changing the pressure of the variable stiffness device

tainly be worth looking into the reason why the calculated and measured results deviate from each other at a pressure of $0.20 \mathrm{MPa}$. For the moment however, results show that it is possible to estimate pantograph compliance when equipped with a variable stiffness devices within the above mentioned range and below the above mentioned margin. The variable stiffness range could be extended if the pressure resistance of the device is improved.

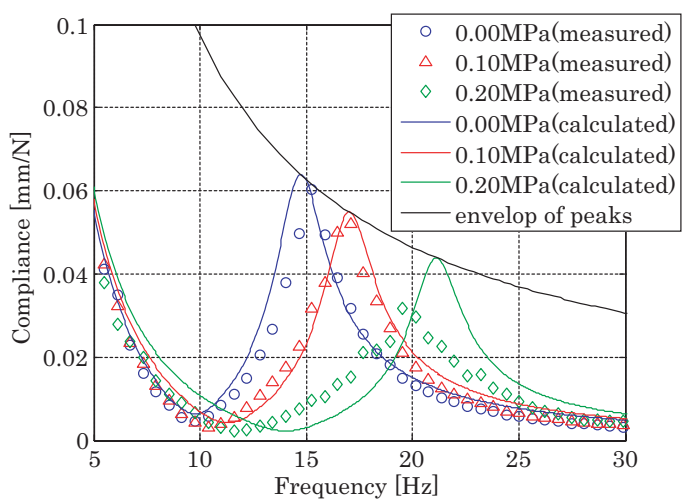

Fig. 12 Estimation of the compliance by means of the stiffness obtained from Fig. 9

\section{Conclusions}

This paper presented a new method for upgrading pantograph performance using variable stiffness devices. To improve contact performance, pan springs, which are typically linear springs, were replaced with variable stiffness springs. The peak frequencies of the compliance characteristics were controlled so as to be consistent with dominant contact wire irregularity frequencies. Results from numerical simulations support the validity of the proposed method. A variable stiffness device prototype was also built which could be installed onto a pantograph. This device is composed of two facing air springs with stiffness which can be controlled by varying the air pressure in the air spring. The ability to control compliance was tested by actual fitting of the device to a pantograph. Results from this experiment indicate that the peak compliance frequency can be shifted by changing the pressure. Currently the peak frequency range which can be controlled is from about $15 \mathrm{~Hz}$ to $20 \mathrm{~Hz}$. It should be possible to extend this range by improving pressure resistance in the variable stiffness device.

\section{References}

[1] G. Galeotti, et. al., "Servo actuated railway pantograph for high-speed running with constant contact force," Proc. IMechE, Vol.207, pp.37-49, 1993.

[2] G. Diana, et. al., "Active Control of High Speed Train Pantographs," Proc. Third International Conference on Motion and Vibration Control, Chiba, Japan, September 1-6, 1996.

[3] D. N. O'Connor, et. al., "Active Control of a High-Speed Pantograph," Journal of Dynamic Systems, Measurement, and Control, Vol.119, pp.1-4, March 1997.

[4] G. Poetsch, et. al., "Pantograph/Catenary Dynamic Control," Vehicle System Dynamics, Vol.28, pp.159-195, 1997.

[5] B. Allotta, et. al., "An active suspension system for railway pantographs: the T2006 prototype," Proc. IMechE, Vol. 223(F), pp.15-29, 2009.

[6] Y. Yamashita and M. Ikeda, "Basic Study on Performance Upgrade of Pantograph Using Variable Stiffness," RTRI Report, Vol.22, No.12, pp.23-28, 2008 (in Japanese). 